m24.p01

\section{Synchrotron-Radiation Computed Laminography - A New Method for the Three-Dimensional Imaging of Flat Objects}

\section{Helfen ${ }^{1,2}, \underline{\text { T. Baumbach }}{ }^{1}$, P. Pernot ${ }^{2}$, P. Mikulik $^{3}$}

${ }^{I}$ ANKA / Institute for Synchrotron Radiation, Forschungszentrum Karlsruhe GmbH, P.O. Box 3640, D-76021 Karlsruhe, Germany. ${ }^{2}$ European Synchrotron Radiation Facility, F-38043 Grenoble, France. ${ }^{3}$ Masaryk University, Kotlarska 2, 61137 Brdno, The Czech Republic.

\section{Keywords: synchrotron radiation imaging, tomography, three-dimensional image reconstruction}

In the paper the methodical development and first instrumental realization of computed laminography with synchrotron radiation is described.

Synchrotron radiation computed Laminography, in the following called SRCL, combines tomosynthesis principles with the specific advantages of synchrotron radiation, such as high brilliance (allowing contrast improvement by working with monochromatic radiation), high partial coherence (enabling to use phase contrast enhancement due to Fresnel diffraction), high spatial resolutions with electronic detector systems with fast read-out and high dynamic range.

$\mathrm{CT}$ consists in the acquisition of a series of projection radiographs for different rotation angles around the tomographic axis, which is set perpendicular to the transmitted beam direction. By applying suitable reconstruction algorithms, a $3 \mathrm{~d}$ image of the sample volume can be reconstructed, providing that the entire sample width was imaged. Since the field of view of digital 2D detectors is limited - CT has to accept a compromise between spatial resolution and maximum lateral object width - a limitation, which often hinders applications of CT for non-destructive testing of micro systems, e.g. the inspection of the bump bonds of flip-chip bonded micro devices.

Contrary to computed tomography, for flat objects the resolution realizable by SRCL does not depend on theobject size. By some simple modification of the scanning geometry and adaptation of tomosynthesis reconstruction algorithms, SRCL enables non-destructively imaging of the 3D-structure of flat, laterally extended objects such as devices of micro system technology. In the talk we discuss the novel instrumental set-up, based on a new concept also compared to laboratory tomosynthesis set-ups. By a series of first experimental examples we show the feasibility of the method for imaging with both, white and monochromatic radiation and high spatial resolution down to submicron scale.

The comparison to phantom samples demonstrates the methodical and instrumental feasibilities; the selected examples illustrate the method's potential for non-destructive testing and quality assurance.

\section{m26.p01}

\section{Crystal Structure of Zr-doped $\mathrm{KTiOPO}_{4}$ crystals at 293 and $105 \mathrm{~K}$ temperature}

Olga Alekseeva $^{a}$, Natalia Sorokina ${ }^{\mathrm{a}}$, Adam Pietrazsko $^{\mathrm{b}}$, Murtazali Rabadanov ${ }^{\mathrm{a}}$, Valentina Voronkova ${ }^{\mathrm{c}}$, Elena Agapova ${ }^{\mathrm{c}}$

${ }^{a}$ Institute of Crystallography RAS, Moscow, Russia. ${ }^{b}$ Institute of Low Temperature and Structural Research PAS, Wroclaw, Poland. ${ }^{c}$ Moscow State University, Moscow, Russia.E-mail: olalex@ns.crys.ras.ru

Keywords: crystal structure analysis, nonlinear optical properties, structure - properties relationships

Crystals of the extensive $\mathrm{KTiOPO}_{4}(\mathrm{KTP})$ family attend much attention because of their excellent non-linear optical properties. Doping of these crystals and studying of their structure have been done in order to find crystals with better non-linear optical properties and also to understand of the structural reasons for these properties. The KTP family counts more than 100 compounds, but only some of them have the improved non-linear properties: crystals with total substitution of $\mathrm{P}$ by As and crystals doped with $\mathrm{Nb}$ (at $\mathrm{Nb}$ content about $4 \%$ ) or with $\mathrm{Zr}$. At that for the crystals doped with $\mathrm{Zr}$ the second harmonic generation intensity is twice as much [1,2]. X-ray structural analysis was done for the first time by as for the crystals KTP:Zr with $1.5 \%$ $\mathrm{Zr}$. The experiment data were received at two temperatures: $\mathrm{T}=293 \mathrm{~K}$ (about 20000 reflexes were registered) and $\mathrm{T}=105$ $\mathrm{K}$ (about 17000 reflexes registered). R-factors were $2.08 \%$ and $1.84 \%$ for the structures at $\mathrm{T}=293$ and $\mathrm{T}=105 \mathrm{~K}$ respectively. Crystal chemistry analysis was done and the structural conditionality of changes of physical properties was revealed for this family of crystals.

[1] Chani V.I., Shimamura K., Endo S., Fukuda T., J. of Crystal Growth. 1997, 171, 472.

[2] Voronkova V.I., Yanovskii V.K., Leontieva I.N., et al., Inorg. mater. 2004, 40, 1505. 